\title{
Papers
}

\section{Expression of the Wilms' tumour gene WT1 in the developing human and in paediatric renal tumours: an immunohistochemical study}

\author{
A K Charles, S Mall, J Watson, P J Berry
}

\begin{abstract}
Aims-The Wilms' tumour gene (WT1) product is expressed during the development of the urogenital system. This study was undertaken to evaluate four anti-WT1 antibodies and use the most specific one to examine the expression of WT1 in formalin fixed, paraffin wax embedded tissues from human embryos, fetuses, and paediatric renal neoplasms.

Methods-The antibodies were assessed on paraffin sections of fetal kidney and by western blotting. Immunohistochemical techniques were optimised and performed on a range of embryonic, fetal, and infant tissues from 35 days post-conception to three months of age, and on a selection of paediatric renal neoplasms.
\end{abstract}

Results-The antibodies tested were found to vary in their specificity. Anomalous expression in smooth muscle was seen with one batch of a commercial polyclonal antibody. WT1 protein was detected in both the metanephros and the mesonephros, the spleen, the gonads, and in the peritoneal mesothelium in fetuses. WT1 was expressed in nuclei and was strongest in the podocytes of fetal kidney. The podocytes of infant glomeruli were also positive. There was focal positive staining in Wilms' tumours, nephrogenic rests, and in a cystic partially differentiated nephroblastoma. Staining of nuclei was seen in one of two rhabdoid tumours of the kidney. No positive staining was seen in other renal tumours.

Conclusions-WT1 is detected readily in formalin fixed material. There were differences in specificity between batches of the polyclonal antibodies used. The distribution of the WT1 gene product in tissues and tumours reflected previous findings with in situ hybridisation studies of WT1 mRNA.

(F Clin Pathol: Mol Pathol 1997;50:138-144)

Keywords: WT1; fetus; Wilms' tumour
The Wilms' tumour gene, WT1, is located at $11 \mathrm{p} 13$ and was identified after germline $\underset{\infty}{\omega}$ cytogenetic abnormalities were found at this $ᄋ$ site in patients with aniridia and Wilms' tumour. ${ }^{1}$ It acts as a tumour suppressor gene, $\check{c}$ and appears to be important in genitourinary $\overparen{D}$ development. ${ }^{2}$ WT1 mRNA transcripts are $\vec{\vartheta}$ expressed only in certain cells, mainly in the developing urinary tract, ${ }^{3}$ but also in mesothelial cells, Sertoli cells, granulosa cells, spinal cord, brain, and spleen. ${ }^{45}$ The WT1 gene product is a $52-54 \mathrm{kDa}$ nuclear protein that has four zinc fingers and acts as a transcriptional regulator, mainly as a repressor but sometimes as an activator. ${ }^{5}$ Its exact function is not clear, but it appears to be involved in the regulation of other growth factors including insulin-like growth factor 2 (IGF2), platelet derived growth factor, colony stimulating factor 1 , insulin growth factor receptor, and retinoic acid receptor $\alpha$ genes. It also interacts with p53, ${ }^{6-8}$ appears to have a role in RNA processing, and is thought to act as an $ᄋ$ anti-oncogene in Wilms' tumour development. $^{910}$

The main role of WT1 is in glomerulogenesis, where it appears to be involved in epithelial differentiation from blastema. ${ }^{11}$ The definitive kidney, the metanephros, develops from paraspinal metanephric mesenchyme by a complex interaction with the ureteric bud. The ureter develops from the distal end of the meso- $\mathscr{D}$ nephric duct. ${ }^{2}$ Blastema develops from metanephric mesenchyme; it condenses and $\overline{0}$ develops into ' $S$ ' form nephrons, which then $\mathbb{D}$ mature into glomeruli and proximal tubules. $\mathbb{D}$ Glomerulogenesis ceases at about 36 weeks of $\frac{2}{0}$ gestation and blastema is usually not seen after this time.

Although cytogenetic abnormalities of the WT1 locus at $11 \mathrm{p} 13$ are uncommon in Wilms tumours, loss of heterozygosity at $11 \mathrm{p} 13$ is seen in $\sim 30 \%$ of sporadic Wilms' tumours. ${ }^{12}$ Mutations of the WT1 gene itself are seen in only $10-20 \%$ of sporadic Wilms' tumours. ${ }^{13}$ This suggests that mutations occurring in adjacent genes or control regions, or possibly inactivation 
by genomic imprinting, are involved in some tumours.

Topographic studies of WT1 mRNA by in situ hybridisation have shown gene expression in developing glomeruli, condensing blastema, and the epithelium of the developing nephron. ${ }^{3}$ In addition, WT1 mRNA has been shown in some Wilms' tumours although not in the stromal component. ${ }^{14}$ The gene product has been demonstrated in some Wilms' tumours by immunohistochemistry, the pattern of expression tending to recapitulate the pattern found in the fetal kidney. ${ }^{514}$ Studies have also reported WT1 protein expression in first trimester fetal kidneys and other tissues, and in some paediatric tumours. ${ }^{15}$

Other loci have been implicated in Wilms' tumours where no WT1 abnormality can be demonstrated. These include $11 \mathrm{p} 15$ (where the candidate gene WT2 is located but not yet identified), $16 \mathrm{q}, 7 \mathrm{p} 15,17 \mathrm{q}$, and others. ${ }^{12} 1617$ The development of Wilms' tumour is almost certainly a multistep process.

Most paediatric renal tumours fall into one of four main groups, which are thought to be histogenetically distinct: Wilms' tumour, nephrogenic rests, cystic partially differentiated nephroblastoma, and cystic nephroma; mesoblastic nephroma, both classical and cellular (atypical) types; clear cell sarcoma of the kidney (bone metastasising renal tumour); and malignant rhabdoid tumour of the kidney. Other tumour types, including adult type renal carcinomas and primitive peripheral neuroectodermal tumours, may also present as primary renal tumours in childhood. ${ }^{18}$

Nephrogenic rests consist of blastema, often with an epithelial component. They are thought to arise from blastema persisting after normal nephrogenesis has ceased, and are found in up to $1 \%$ of neonatal kidneys at postmortem examination. ${ }^{19}$ Microscopic rests are associated with $30-40 \%$ of Wilms' tumours in some series, and are believed to be precursors of Wilms' tumour. In these cases they are usually found as a better differentiated zone at the edge of the tumour but also may be found separate from the main tumour. Multiple nephrogenic rests (nephroblastomatosis) may involve both kidneys and indicate a risk of bilateral Wilms' tumour. This association may be seen in patients with a family history of Wilms' tumour, ${ }^{20}$ and in patients with Wilms' tumour associated syndromes such as Drash (Wilms' tumour, glomerulopathy, and ambiguous genitalia in males), WAGR (Wilms' tumour, aniridia, genital abnormalities, and mental retardation), and Beckwith syndrome (macrosomia, omphalocele, neonatal hypoglycaemia, and an increased risk of tumours including Wilms'). ${ }^{19}$ Nephrogenic rests are also associated with cystic renal dysplasia, when their malignant potential appears to be low. ${ }^{21}$

The recent availability of antibodies to the WT1 gene product for use with formalin fixed material prompted this systematic study of the specificity of these antibodies and the expression of WT1 in the major organs of developing embryos and fetuses from 35 days postconception until term, and in paediatric tumours. These tumours included Wilms' tumour, cystic partially differentiated nephroblastoma, nephrogenic rests, mesoblastic nephroma, malignant rhabdoid tumour of the kidney, clear cell sarcoma of the kidney, renal cell carcinoma, and adrenal neuroblastoma. Neuroblastomas were examined as they can be clinically difficult to distinguish from primary renal tumours, and may present as an intrarenal tumour. Our purpose was to document WT1 gene expression in the normal developing kidney, and in renal tumours, and to see whether it was diagnostically useful in the differential diagnosis of paediatric renal tumours.

In the light of initial studies a further selection of non-renal tumours, derived from tissues found positive for WT1 in fetuses, was examined also.

\section{Materials and methods}

Two batches of commercial antibody directed against the WT1 protein, WT C19 (Santa Cruz, California, USA; catalogue number sc-192, batches 6063 and D124), and two antibodies produced by immunising rabbits with the WT1 fusion protein available in the Cancer and Leukaemia in Childhood funded laboratory, University of Bristol were assessed. All were polyclonal antibodies raised in rabbits.

Formalin fixed, paraffin wax embedded kidney from an 18 week fetus was used in the initial assessment of the specificity of each antibody.

Western blotting was performed using WT1 protein obtained from an adenovirus transformed human fetal kidney line T5A $1 .^{22}$ This is derived from a 293 cell line with inducible WT1 expression following transfection with a metallothionine inducible vector (pMEP4).

Postmortem and surgical tissues came from the files of the department of paediatric pathology, Bristol Children's Hospital. Parental consent had been given for using postmortem tissues for research. Serial sections from two well preserved embryos of 35 and 43 days post-conceptional age were selected to include the genitourinary ridge. Sections from a male and a female well preserved normal fetus of 12-13 weeks, 17-18 weeks, 24-25 weeks, and 38-40 weeks gestational age were chosen to include all major organs. Kidneys from two infants (three months old) were also examined.

Thirty one paediatric renal tumours were chosen to include 14 Wilms' tumours (13 renal and one extrarenal) one with focal anaplasia, one cystic partially differentiated nephroblastoma, six nephrogenic rests, four mesoblastic nephromas, two malignant rhabdoid tumours of the kidney, two clear cell sarcomas of the kidney, and two renal cell carcinomas. Two of the Wilms' tumours were later rejected because of insufficient viable tumour and in another case a nephrogenic rest was no longer present in recut sections tested.

In the light of the initial results several examples of tumour types thought to arise from tissues that were positive for WT1 in fetuses were studied. These were three adenomatoid 
A

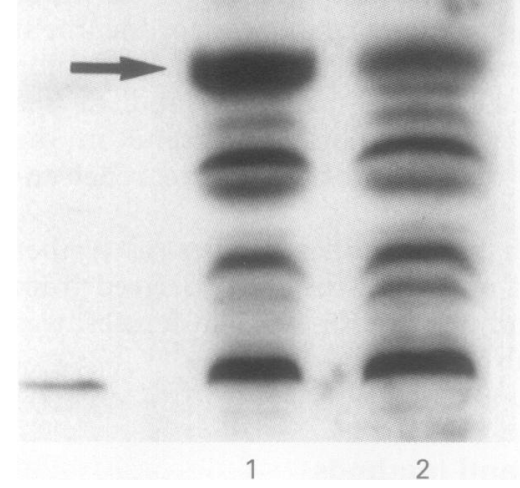

B

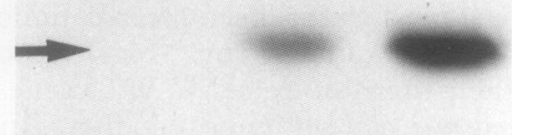

Figure 1 Western blotting. (A) First batch of anti-WT1 antibody C19 (Santa Cruz) showing that WT1 (arrow) is induced in T5A1 (first column, 1) compared with the non-induced T5A1 (second column, 2). Additional bands are clearly seen. (B) Second batch of $C 19$ showing that the WT1 band is clearly increased in the induced T5A1 (second column, 4) compared with the non-induced T5A1 (first column, 3). This antibody appears to be more specific.

Table 1 The expression of the WT1 protein in embryonic and fetal tissues as assessed by C19 antibody

\begin{tabular}{|c|c|c|c|c|c|c|}
\hline Tissue & $\begin{array}{l}\text { Embryo } \\
\text { (7 weeks) }\end{array}$ & $\begin{array}{l}\text { Fetus } \\
\text { (12 weeks) }\end{array}$ & $\begin{array}{l}\text { Fetus } \\
\text { (18 weeks) }\end{array}$ & $\begin{array}{l}\text { Fetus } \\
\text { (25 weeks) }\end{array}$ & $\begin{array}{l}\text { Fetus } \\
\text { ( } 38 \text { weeks) }\end{array}$ & $\begin{array}{l}\text { Infant } \\
\text { (3 months) }\end{array}$ \\
\hline Heart & - & - & - & - & - & - \\
\hline Ling & & - & - & - & - & \\
\hline Bowel & - & - & - & - & - & - \\
\hline Liver & - & - & - & - & - & \\
\hline Pancreas & - & - & & - & - & \\
\hline Kidney & +++ & +++ & ++ & ++ & + & + \\
\hline Testis & Indeter- & - & - & + & - & - \\
\hline Ovary & $\begin{array}{l}\text { minate } \\
\text { gonad }+^{\star}\end{array}$ & - & + & - & - & - \\
\hline Uterus & & - & + & - & - & \\
\hline Spleen & & & + & - & - & - \\
\hline Thymus & & - & - & - & - & \\
\hline Adrenal & & - & - & - & - & \\
\hline Parathyroid & & & - & - & & \\
\hline Pituitary & & & - & & & \\
\hline \multicolumn{7}{|l|}{ Skeletal } \\
\hline muscle & - & - & - & & & \\
\hline Bone & - & - & & & & \\
\hline Brain & - & - & - & - & - & \\
\hline Mesothelium & + & + & + & - & - & \\
\hline Placenta & - & - & - & & - & \\
\hline Decidua & & & ++ & + & - & \\
\hline
\end{tabular}

-, negative staining; blank, not tested; + to +++ , weak to strong positive staining.

$\star$ The morphology of testis and ovaries are very similar at this gestational age.

tumours, three granulosa cell tumours, an ovarian mucinous cystadenoma, and two thoracic mesotheliomas.

The tissues were all formalin fixed, processed into paraffin wax, sectioned at $4 \mu \mathrm{m}$, placed on 3-amino-propyltriethoxysilane (APES) coated slides and baked for at least one hour at $60^{\circ} \mathrm{C}$. The immunohistochemical method was optimised by varying the length of time of microwave antigen retrieval using a citrate buffer $(2.1 \mathrm{~g}$ citric acid in distilled water, $\mathrm{pH}$ 6.0). Slides were placed in a $500 \mathrm{ml}$ covered tray, heated on high power, and topped up with distilled water after 20 minutes. Antibodies were titrated with sections of normal fetal kidney using the avidin-biotin method and peroxidase with diaminobenzidine

(DAB) as the detection system. The optimal dilution for the $\mathrm{C} 19$ antibody was $1 / 1000$ in phosphate buffered saline.

An 18 week fetal kidney was used as a positive control in each run. A duplicate slide of each case omitting the primary antibody was processed as a negative control. Only nuclear staining was scored positively. Cytoplasmic staining sometimes seen in mature proximal tubules was regarded as an artefact due to incompletely blocked endogenous peroxidase, and was present in the negative control sections.

\section{Results}

IMMUNOHISTOCHEMICAL ASSESSMENT OF THE FOUR ANTIBODIES ON FETAL KIDNEY

The best results were obtained using the two batches of commercial antibody (Santa Cruz). Although reasonable results had been obtained previously on frozen sections using the other antibodies, these produced a high background signal on paraffin wax embedded sections. The optimal pretreatment time for microwave retreival was between 20 and 40 minutes, with weak staining at 10 minutes, and morphological deterioration at an hour. Therefore, 40 minutes was used in subsequent tests.

\section{WESTERN BLOTTING}

Western blotting showed considerable differences between the two batches of $\mathrm{C} 19$ antibody. The first batch showed strong staining of nonWT1 bands, the second batch (batch D124) was more specific for WT1 (fig 1). This difference in specificity may explain the positive staining of muscle nuclei seen with the first antibody.

IMMUNOHISTOCHEMICAL ASSESSMENT OF THE ANTIBODIES ON EMBRYONIC AND FETAL TISSUES The two batches of antibody from Santa Cruz were evaluated further on a selection of fetal tissues and tumours. The first batch showed reaction with smooth muscle and cardiac muscle nuclei, as well as the expected pattern in kidney. No skeletal muscle staining was seen. The second batch did not show staining of smooth muscle. The results reported in the rest $\frac{7}{0}$ of this paper are with the second antibody, although small nephrogenic rests already ex- o amined with the first antibody were not $N$ restained in order to conserve irreplaceable N archival tissue. The findings are shown in table 1 , and are summarised below.

\section{Kidney}

Kidney was positive at all gestational ages, showing a characteristic staining pattern (fig $2 \mathrm{~A})$. In the two embryos there was clear positivity in nuclei of both the mesonephric $\stackrel{\mathbb{Q}}{\Omega}$ glomeruli and metanephros (fig 2B). Moderately strong but clear staining was seen in the proximal part of ' $S$ ' form nephrons. Weaker staining was seen in the condensing blastema adjacent to developing glomeruli in the superficial cortex. The strongest reaction was seen in podocytes covering the glomerular tuft. The parietal epithelial cells lining Bowman's capsule also showed some positivity and an abrupt transition to the negatively staining proximal tubule. Staining of the podocytes persisted 


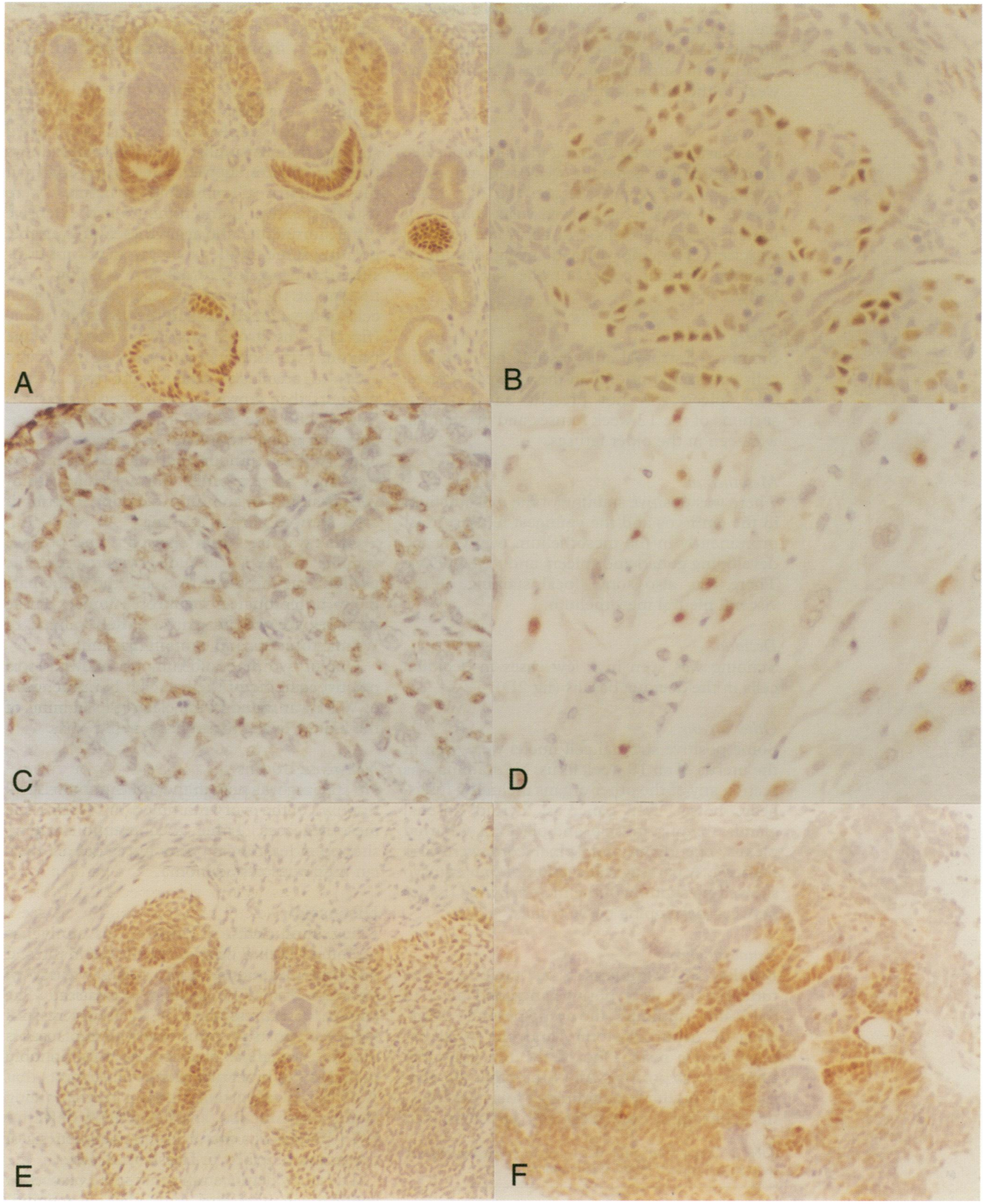

Figure 2 Immunohistochemical detection of WT1 protein. (A) Kidney at 18 weeks'gestation showing strong staining of the nuclei of glomerular podocytes, and weaker staining of the ' $S$ ' form nephrons and the condensing blastema. (B) Mesonephric glomeruli of an embryo showing positive staining of the mesonephric podocyte nuclei. (C) Ovary at 18 weeks' gestation showing staining of the stromal cells and strong staining of the mesothelium (top left of the figure). (D) Decidua from an 18 week gestation pregnancy showing positively staining decidual cells and negatively staining trophoblast cells.

(E) Wilms'tumour showing positive staining of the nuclei in certain areas of the blastema and some tubules. Note the stroma is negative, and that the most positive areas of blastema tend to be at the edge of the blastemal islands. (F) Wilms'tumour showing nuclear staining of most, but not all, of the tubules and some areas of the blastema. This mimics the pattern seen in the developing glomerulus and proximal tubule. 
after 36 weeks' gestation when nephrogenesis has ceased, and staining was seen in normal glomeruli in the kidney adjacent to the renal tumours in surgical specimens. Staining was weak in the infant kidneys .

\section{Gonads}

The ovaries showed staining in granulosa cells, although it was generally weak and only seen strongly in the youngest fetuses (fig 2C). Testis from a 25 week fetus showed weak staining of immature Sertoli cells. No germ cell staining was seen. The embryos had strong staining of gonadal stromal cells, but the sex was not known.

\section{Spleen}

Staining of stromal and possible haemopoetic cells was seen, but only in the 18 week fetuses. The spleen was not present on the recut sections of the 12 week fetuses and no staining was seen in the older fetuses.

\section{Mesothelium}

There was positive staining of the mesothelium in the embryos and the youngest fetuses, most prominently in the mesothelium overlying the developing gonad and spleen, and in the pelvis. There was also some focal staining of the visceral pleural mesothelium.

\section{Decidua}

Staining was seen in a few cases in decidual cells in the decidua basalis (fig $2 \mathrm{D}$ ).

\section{Other organs}

Some positive stromal cell nuclei were seen in the uterus of an 18 week fetus. No staining was seen in any of the other organs including lungs, heart, bowel, liver, pancreas, thyroid, adrenal glands, thymus, muscle, and placenta. Choroid plexus, ependyma and spinal cord were also negative. A parasagittal section of one embryo showed no staining of the mesenchymal tissues between developing nerve trunks.

\section{Renal tumours}

Results are summarised in table 2. The tumours that were positive included most of the Wilms' tumours, the cystic partially differentiated nephroblastoma, and the perilobar nephrogenic rests. One of the two rhabdoid tumours was positive. All of the other paediatric renal tumours, including all four mesoblastic nephromas, both clear cell sarcomas, and

Table 2 Expression of the WT1 protein in paediatric renal tumours as assessed by the C19 antibody

\begin{tabular}{lc}
\hline Tumour & Number positive \\
\hline Wilms' tumour & $10 / 12$ \\
Nephrogenic rests & $4 / 4$ \\
$\quad$ Perilobar & $0 / 1$ \\
$\quad$ Intralobar & $1 / 1$ \\
Cystic partially differentiated & \\
$\quad$ nephroblastoma & $0 / 2$ \\
Mesoblastic nephroma & $0 / 2$ \\
$\quad$ Classical & $0 / 2$ \\
$\quad$ Cellular & $1 / 2$ \\
Clear cell sarcoma of the kidney & $0 / 2$ \\
Rhabdoid tumour of the kidney & $0 / 2$ \\
Renal cell carcinoma & \\
Neuroblastoma & \\
\hline
\end{tabular}

the two neuroblastomas were negative. Adjacent kidney, if present, showed staining of the podocytes, forming an internal positive control.

The pattern of staining in the Wilms' tumours varied from one case to another. Some tumours showing glomerulogenesis often had a staining pattern similar to that in fetal kidneys (fig 2E). Others showed zones of positivity in the blastema. Glomeruloid structures were usually positive. Some tumours had extensive tubule formation, and again these were variably positive, some tubules showing an abrupt transition from positive to negative staining reminiscent of the abrupt change in the parietal cells of the glomerulus and the proximal tubule (fig $2 \mathrm{~F}$ ). The stroma was always negative. One tumour with focal anaplasia was negative in the areas of anaplasia. One of the two Wilms' tumours that showed no positive staining was from a child with WAGR syndrome; the associated intralobar rest was also negative. The other Wilms' tumour that stained negatively was from an apparently normal child. Uninvolved kidney in the same sections from both cases showed normal staining.

Other tumours that showed some positive staining for WT1 included the microscopic perilobar nephrogenic rests and the cystic partially differentiated nephroblastoma. These tumours are thought to be closely related to Wilms' tumours and contain blastema.

One of two rhabdoid tumours showed focal positive staining of their nuclei. No expression of WT1 was found in the clear cell sarcomas of the kidney or the mesoblastic nephromas.

\section{Non-renal tumours}

No staining was seen in the three granulosa cell tumours of the ovary examined, or two thoracic mesotheliomas. Three adenomatoid tumours showed at least focal positivity, as did an ovarian mucinous cystadenoma.

\section{Discussion}

Using immunohistochemistry, this study has examined both a wide range of fetal tissues at $\frac{D}{O}$ different gestational ages, and a variety of tumours, for WT1 protein expression. The o pattern of expression of WT1 found in fetal N kidney supports previous observations, ${ }^{34}$ and is N present throughout glomerulogenesis. Staining of podocytes in mature glomeruli suggests that WT1 has an important function in maintaining glomerular function, and presumably defective $\mathscr{f}$ WT1 protein is a factor in the pathogenesis of 70 the glomerulopathy in Drash syndrome.

The study demonstrates that some batches of commercial polyclonal antibodies give signifi- $\mathbb{D}$ cant differences in staining: muscle staining seen with one batch of antibody was not seen with 8 another batch. Although muscle staining has been described in another study ${ }^{15}$ that also used an antibody from Santa Cruz, mRNA for WT1 has not clearly been demonstrated by in situ hybridisation techniques in muscle, although expression in the somites of vertebrate embryos has suggested a role for WT1 in smooth, cardiac, and skeletal muscle development. ${ }^{23}$ In contrast, muscle differentiation in Wilms' tumours is 
associated with WT1 mutations. ${ }^{24}$ Ramani and Cowell found that the positive staining in muscle was blocked by pretreatment with the immunising peptide, ${ }^{15}$ suggesting that muscle may have a nuclear protein with a similar epitope to WT1.

The staining pattern in the tumours was in agreement with previous studies. ${ }^{14}$ The pattern found in Wilms' tumours generally recapitulated the pattern of expression found in the fetal kidney. Some areas of blastema, particularly adjacent to the stroma or epithelium, often showed positive staining. Not all epithelial structures were positive and the sudden transition of some tubules appeared to be similar to the transition between expression of WT1 in the Bowman's capsule and absence of WT1 in the proximal tubules. No positive staining was seen in the stroma of the tumours.

One of the two Wilms' tumours that showed no staining was from a patient with WAGR syndrome and a cytogenetic deletion at $11 \mathrm{p} 13$ indicating loss of one allele of WT1 and the nearby gene(s) associated with aniridia. In WAGR patients a further smaller genetic loss (the second hit) causes functional impairment of the remaining WT1 gene, resulting in development of Wilms' tumour. This may have caused the loss of the epitope recognised by the antibody in this patient's tumour, explaining the lack of staining.

Although the other WT1 negative Wilms' tumour had areas that would be expected to be positive in the light of expression in other tumours, negative staining could not be attributed to poor fixation because positive staining of adjacent uninvolved kidney was seen. This case also had an intralobar nephrogenic rest known to be associated with cytogenetic abnormalities of the $11 \mathrm{p} 13$ area and WT1 mutations in many cases. However, there were no syndromic features or evidence of bilateral disease to suggest a somatic mutation of WT1 in this second patient.

Approximately $10 \%$ of sporadic Wilms' tumours show WT1 mutations, and may therefore lack WT1 expression. The proportion of negatively staining Wilms' tumours in our series is in keeping with this generally accepted figure.

In their recent paper, Ramani and Cowell found positive staining for WT1 in all their Wilms' tumours including those with anaplasia. ${ }^{15}$ We had a single case with focal anaplasia that was focally positive in the non-anaplastic areas, but negative in anaplastic cells. ${ }^{15}$ The reason for this disparity is not clear, but, generally, tumours showed WT1 staining only in some areas of blastema and epithelium. Anaplasia may have arisen from parts of the tumour showing no WT1 expression.

Positive staining for WT1 in one of two rhabdoid tumours was of interest. Other genes expressed in Wilms' tumours have been described in rhabdoid tumours, including IGF2. ${ }^{25}$ This particular tumour was otherwise typical of rhabdoid tumours and did not show any features of Wilms' tumour. The histogenesis of malignant rhabdoid tumours of the kidney is unknown, but they may develop from undifferentiated nephrogenic mesenchyme, ${ }^{26}$ which could explain the variable presence of WT1.

Controls showed no positive staining except for cytoplasmic staining of the liver, and the proximal tubule of the kidney, owing to the presence of endogenous biotin. No clear nuclear staining was seen.

Following these initial results some other tumours thought to arise from tissues that were positive in the fetuses or embryos were examined for WT1 expression. A mucinous cystadenoma of the ovary and two adenomatoid tumours (of the uterus and Fallopian tube, respectively) showed focal positive staining. We had found strong expression of WT1, as assessed by immunohistochemistry, in the mesothelium of the pelvis in embryos, and both of these tumours are thought to be derived from mesothelium. Two thoracic malignant mesotheliomas from adults were negative, but a recent paper examining mesotheliomas has demonstrated that WT1 may be useful in identifying these tumours. ${ }^{27}$ We have recently demonstrated that WT1 is seen in the desmoplastic small round cell tumour, which has a translocation involving WT1 and the Ewing sarcoma gene. ${ }^{28}$ This tumour is closely associated with mesothelium, and shows immunohistochemical evidence of epithelial differentiation.

\section{Conclusion}

WT1 protein expression may be identified in formalin fixed tissue using immunohistochemistry, but care needs to be taken to evaluate unexpected staining with polyclonal antibodies. WT1 is not seen in all Wilms' tumours and may be present in a range of tumours derived from the urogenital tract or mesothelium: tissues that express WT1 in the embryonic and fetal period. Therefore, anti-WT1 antibodies appear to have limited diagnostic use, but are a helpful tool in studying the relationship of morphology to the expression of the WT1 protein in a range of paraffin wax embedded fixed tissues.

AK Charles was supported by a Smith and Nephew Research Fellow grant during part of this study and J Watson was funded by the Medical Research Council. S Mall is supported by the Foundation for the Study of Sudden Infant Death.

1 Franke U, Holmes LB, Atkins L, Riccardi VM. AniridiaWilms' tumor association: evidence for specific deletion of Wilms tumor association: evidence for specific

2 Bard JBL McConnell JE, Davies JA. Towards a genetic basis for kidney development. Mech Dev 1994;48:3-11.

3 Pritchard-Jones K, Fleming S, Davidson D, Bickmore W, Porteous D, Gosden C, et al. The candidate Wilms' tumour gene is involved in genitourinary development. Nature 1990;346:194-7.

4 Pelletier J, Schalling M, Buckler AJ, Rogers A, Haber DA, Housman D. Expression of the Wilms' tumor gene WT1 in the murine urogenital system. Genes Dev 1991;5:1345-56.

5 Huang A, Campbell CE, Bonetta L, McAndrews-Hill MS, Chilton-McNeil S, Coppes MJ, et al. Tissue, developmental, and tumor-specific expression of divergent transcripts

6 Wang ZY, Qiu QQ, Gurrieri M, Huang J, Deuel TF. WT1, the Wilms' tumor suppressor gene product, represses transcription through an interactive nuclear protein. Oncogene 1995;10:1243-7.

7 Goodyer P, Dehbi M, Torban E, Bruening W, Pelletier J. Repression of the retinoic acid receptor-alpha gene by the Wilms' tumor suppressor gene product, WT1. Oncogene 1995;10:1 125-9.

8 Maheswaran S, Park S, Bernard A, Morris JF, Rauscher FJ 3rd, Hill DE, et al. Physical and functional interaction 1993;90:5100-4. 
9 Larsson SH, Charlieu JP, Miyagawa K, Engelkamp D, Rassoulzadegan M, Ross A, et al. Subnuclear localization of WT1 in splicing or transcription factor domains is regulated by alternative splicing. Cell 1995;81:391-401.

10 Haber DA, Buckler AJ, Glaser T, Call KM, Pelletier J, Sohn $\mathrm{RL}$, et al. An internal deletion within an $11 \mathrm{p} 13$ zinc finger gene contributes to the development of Wilms' tumor. Cell 1990;61:1257-69.

11 Kreidberg JA, Sariola H, Loring JM, Maeda M, Pelletier J, Housman D, et al. WT-1 is required for early kidney development. Cell 1993;74:679-91.

12 Coppes MJ, Bonetta L, Huang A, Hoban P, ChiltonMacNeill S, Campbell CE, et al. Loss of heterozygosity mapping in Wilms' tumor indicates the involvement of hree distinct regions and a limited role for nondisiunction three distinct regions and a limich role for non or mitotic recombina 5.326-34.

Brown KW, Wilmore HP, Watson JE, Mott MG, Berry PJ, Maitland NJ. Low frequency of mutations in the WTI coding region in Wilms' tumor. Genes Chromosomes Cance 1993;8:74-9.

14 Pritchard-Jones K, Fleming S. Cell types expressing the Wilms' tumour gene (WT1) in Wilms' tumours: implications for tumour histogenesis. Oncogene 1991;6:2211-20.

15 Ramani P, Cowell JK. The expression pattern of Wilms' tumour gene (WT1) product in normal tissues and paediatric renal tumours. F Pathol 1996;179:162-8.

16 Grundy PE, Telzerow PE, Breslow N, Moksness J, Huff V, Paterson MC. Loss of heterozygosity for chromosomes $16 \mathrm{q}$ and $1 \mathrm{p}$ in 'Wilms' tumors predicts an adverse outcome. and lp in Wilms' tumors

17 Wilmore HP, White GF, Howell RT, Brown KW. Germline and somatic abnormalities of chromosome 7 in Wilms tumor. Cancer Genet Cytogenet 1994;77:93-8.

18 Murphy WM, Beckwith JB, Farrow GM. Tumors of infancy and childhood. In: Tumors of the kidney, bladder, and related urinary structures. Bethesda: Armed Forces Institute of Pathology, 1994:12-91.

19 Beckwith JB, Kiviat NB, Bonadio JF. Nephrogenic rests, nephroblastomatosis, and the pathogenesis of Wilms' nephroblastomatosis, and the pathogenesis

20 Breslow NE, Olson J, Moksness J, Beckwith JBB, Grundy P. Familial Wilms' tumor: a descriptive study. Med Pediatr Oncol 1996;27:398-403.

21 Gaddy CD, Gibbons MD, Gonzalez ET, Finegold MJ. Obstructive uropathy, renal dysplasia and nodular renal blastema: is there a relationship to Wilms' tumour? f Urol 1985;134:330-3

22 Malik KT, Poirier V, Ivins SM, Brown KW. Autoregulation of the human WT1 gene promoter. FEBS Lett 1994;349: 75-8.

23 Kent J, Coriat AM, Sharpe PT, Hastie ND, van Heyningen $\mathrm{V}$. The evolution of WT1 sequence and expression pattern in the vertebrates. Oncogene 1995;11:1781-92.

24 Miyagawa K, Kent J, Schedl A, van Heyningen V, Hastie ND. Wilms' tumour - a case of disrupted development. $\mathcal{F}$ Cell Sci 1994;18(Suppl): $1-5$.

25 Sharifah NA, Yun K. Malignant rhabdoid tumor of the kidney expresses insulin-like growth factor II transcripts. Pathology 1994;26:134-7.

26 Weeks DA, Beckwith JB, Mierau GW, Luckey DW. Rhabdoid tumour of kidney. Am f Surg Pathol 1989;13: 439-58.

27 Amin KM, Litzky LA Smythe WR, Mooney AM, Morris JM, Mews DJ, et al. Wilms' tumor 1 susceptibility (WT1) JM, Mews DJ, et al. Wilms tumor 1 susceptibility (WT1) gene products are selectively expressed in

28 Charles AK, Moore IE, Berry PJ. Immunohistochemical detection of the Wilms' tumour gene WT1 in desmoplastic small round cell tumour. Histopathology 1997;30:31214. 